Check for updates

Cite this: RSC Adv., 2018, 8, 37165

Received 9th October 2018

Accepted 29th October 2018

DOI: $10.1039 / \mathrm{c} 8 \mathrm{ra0} 0373 \mathrm{~h}$

rsc.li/rsc-advances

\section{Microwave-assisted facile synthesis of poly(luminol-co-phenylenediamine) copolymers and their potential application in biomedical imaging $\dagger$}

\begin{abstract}
Ufana Riaz, (DD *a Sapana Jadoun, ${ }^{\text {a }}$ Prabhat Kumar, ${ }^{b}$ Raj Kumar ${ }^{\mathrm{c}}$ and Nitin Yadav ${ }^{\mathrm{d}}$
Conjugated copolymers have attracted much attention because of their outstanding photo-physical properties. The present work reports for the first time, microwave-assisted copolymerization of $\mathrm{O}^{-}$ phenylenediamine with luminol using different weight ratios of the two monomers. The composition of the copolymers was confirmed by Fourier transform infrared spectroscopy (FTIR) and nuclear magnetic resonance spectroscopy $\left({ }^{1} \mathrm{H}-\mathrm{NMR}\right)$ while monomer reactivity ratios were determined using the Fineman-Ross method. Ultraviolet-visible spectroscopy revealed the variation in polaronic states upon copolymerization while X-ray diffraction (XRD) and transmission electron microscopy (TEM) analyses showed the morphology of the copolymers to be intermediate between that of the homopolymers. Confocal analysis and fluorescence studies revealed that the copolymers showed composition based blue as well as red emission which could be utilized for in vivo imaging of cancer cells.
\end{abstract}

\section{Introduction}

Stimuli responsive fluorescent nanomaterials such as quantum dots, (QDs), ${ }^{1,2}$ carbon nano-dots (CDs), ${ }^{3,4}$ polymer $\operatorname{dots}^{5,6}$ etc. have been extensively investigated for their biological applications particularly in the detection and diagnosis of tumours at early stages of occurrence. QDs are well-known for their remarkable optoelectronic properties, size-tunability, high quantum yield (QY), outstanding resistance to photo-bleaching, and exceptionally large Stokes shift. ${ }^{7,8}$ However, most of the QDS that are effectively used as contrast imaging agents are toxic inorganic nanoparticles, which raises serious concerns regarding their potential long-term cytotoxicity under in vivo conditions. ${ }^{9-12}$ Hence, the quest for alternative biocompatible as well as non-cytotoxic materials has gained momentum.13,14 Several near infrared (NIR) light-emitting polymers such as polyfluorenes (PFs), ${ }^{15}$ polyethylene-dioxythiophene (PEDOT), ${ }^{\mathbf{1 6}}$ polypyrrole (PPy) ${ }^{17}$ etc. have been demonstrated to exhibit good biocompatibility in a number of in vitro and in vivo studies. However, efforts are still underway to develop effective

${ }^{a}$ Materials Research Laboratory, Department of Chemistry, Jamia Millia Islamia, New Delhi-110025, India.E-mail: ufana2002@yahoo.co.in

${ }^{b}$ Advanced Instrumentation Research Facility, Jawaharlal Nehru University, New Delhi-110067, India

${ }^{c}$ Cancer and Radiation Therapeutics Laboratory, School of Life Sciences, Jawaharlal Nehru University, New Delhi-110067, India

${ }^{d}$ Department of Chemistry, Indian Institute of Technology, Delhi-110016, India

$\dagger$ Electronic supplementary information (ESI) available. See DOI: 10.1039/c8ra08373h conjugated polymer based theranostic agents for controllable cancer detection and diagnosis. Applications of aggregationinduced emission (AIE) of luminogens such as cyanosubstituted diarylethene derivatives, ${ }^{18,19}$ tetraphenylethene (TPE) derivatives, ${ }^{20,21}$ distyrylanthracene derivatives, ${ }^{22,23} \alpha$-cyanostilbene derivatives ${ }^{\mathbf{2 4 , 2 5}}$ in chemosensors and bio-imaging are well-documented. $^{26}$ Polymerization-induced self-assembly (PISA) has also been investigated to prepare positive contrast agents for magnetic resonance imaging (MRI). ${ }^{27,28}$

Near infrared emission (NIR) provides high sensitivity towards in vivo measurements through minimum interference of auto-fluorescence from biological tissues and hence is regarded as a desirable technology for the diagnostic bioimaging. Hence, aith the view to develop a tunable bioimaging agent, we have for the first time, attempted to copolymerize luminol with POPD via microwave-assisted chemical polymerization method. Among the several polymerization techniques known, microwave-assisted synthesis offers several advantages such as solid phase synthesis, increased reaction rates, and improved product yields. $^{29,30}$

Luminol was chosen as a co-monomer because it is commonly used as a chemiluminescence reagent in forensic medicine for selective detection of blood stains, but no work has been reported on the chemical copolymerization of luminol. ${ }^{31-34}$ $O$-phenylenediamine (OPD) on the other hand, is an extensively explored polyaniline (PANI) derivative which exhibits outstanding photo-physical properties and fairly good water solubility. ${ }^{35,36}$ Copolymerization of the two monomers was carried out with a view to tailor the opto-electronic and 
electrochemical properties of copolymers by varying the comonomer ratio. ${ }^{37-39}$

The copolymers were analysed for their spectral and morphological characteristics by employing infrared (IR), ${ }^{1} \mathrm{H}$ NMR, ultraviolet-visible (UV-Vis) spectroscopies, X-ray diffraction (XRD) and transmission electron microscopic (TEM) techniques. Fluorescence spectroscopy and confocal imaging were also carried out to explore the emission characteristics of the copolymers. Spectral studies confirmed the formation of a random copolymer exhibiting composition dependent optical characteristics. Cell viability was investigated using the methyl tetrazolium (MTT) assay and human cervical tumour (HeLa) cells. The imaging capability of the copolymers was analysed via live cell imaging studies.

\section{Experimental}

Luminol, o-phenylenediamine, dimethyl sulfoxide and 3(4,5dimethyl thiozol-2-yl)-2,5-diphenyl tetrazolium bromide) (MTT) assay were purchased from Sigma Aldrich, USA and were used without further purification. RPMI-1640, FBS, trypsin and antibodies were purchased from GIBCO Grand Island, New York, USA.

\section{Microwave-assisted synthesis of homopolymers of $o$ - phenylenediamine and luminol}

$O$-phenylenediamine (OPD) $\left(1 \mathrm{~g}, 9 \times 10^{-2} \mathrm{~mol}\right)$ was dispersed in deionized water $(50 \mathrm{ml})$ in an Erlenmeyer flask $(100 \mathrm{ml})$ containing benzoyl peroxide (BPO) $\left(4.47993 \mathrm{~g}, 2.5 \times 10^{-1} \mathrm{~mol}\right)$. The reaction mixture was exposed to microwave irradiation for 15 min at $25{ }^{\circ} \mathrm{C}$ in microwave oven (model LBP125-230, Ladd Research (USA)) as per synthesis conditions reported in our previous studies. ${ }^{39}$ The obtained product was then kept overnight in a deep freezer at $-10{ }^{\circ} \mathrm{C}$ and was centrifuged after $24 \mathrm{~h}$ with distilled water to remove the unreacted impurities. The synthesized polymer was dried at $70{ }^{\circ} \mathrm{C}$ for $72 \mathrm{~h}$ in vacuum oven to ensure complete removal of moisture, unreacted monomer and other volatile impurities. The polymerization of luminol (PLU) was carried out in a similar manner using luminol $(1 \mathrm{~g}, 5$ $\left.\times 10^{-2} \mathrm{~mol}\right)$ and BPO (4.47993 g, $\left.2.5 \times 10^{-1} \mathrm{~mol}\right)$. The yields obtained for POPD and PLU were $67.75 \%$ and $84.33 \%$ respectively.

\section{Synthesis of copolymers of $o$-phenylenediamine and luminol}

To prepare copolymers of POPD : PLU, monomer (OPD) $(0.5 \mathrm{~g}, 5$ $\left.\times 10^{-2} \mathrm{~mol}\right)$ and luminol (LUM) $\left(0.2 \mathrm{~g}, 1 \times 10^{-2} \mathrm{~mol}\right)$ were added to Erlenmeyer flask (100 ml) containing BPO (4.47993 g, $2.5 \times 10^{-1} \mathrm{~mol}$ ) homogeneously dispersed in distilled water (50 $\mathrm{ml}$ ). The reaction mixture was exposed to microwave irradiation for $15 \mathrm{~min}$ at $25^{\circ} \mathrm{C}$ in microwave oven (model LBP125-230, Ladd Research (USA)) as per synthesis conditions reported in our previous studies. ${ }^{39}$ The synthesized copolymer centrifuged with distilled water and removal of iron was ensured by testing the filtrate with potassium ferrocyanide. ${ }^{39}$ The obtained copolymer was dried in vacuum oven at $70{ }^{\circ} \mathrm{C}$ (for $72 \mathrm{~h}$ ) to ensure complete removal of water and other volatile impurities. The copolymer was designated as POPD/PLU-80/20. Similarly, the synthesis of other POPD : PLU copolymers were carried out by varying the co-monomer ratios OPD : LUM as $0.3 \mathrm{~g}, 2 \times 10^{-2}$ mol: $0.5 \mathrm{~g}, 3 \times$ $10^{-2} \mathrm{~mol}$ and $0.25 \mathrm{~g}, 2 \times 10^{-2}$ mol: $1.63 \mathrm{~g}, 1 \times 10^{-2} \mathrm{~mol}$ respectively. The synthesized copolymers were designated as POPD/PLU-50/50 and POPD/PLU-20/80. The percent yield obtained for the copolymers was POPD/PLU-80/20: 75.03\%; POPD/ PLU-50/50: $78.27 \%$ and POPD/PLU-20/80: 79.81\%.

\section{Characterization}

The viscosity average molar mass was investigated by viscosity method as reported in our previous studies. ${ }^{39}$ FT-IR spectra were recorded on FT-IR spectrophotometer model Shimadzu IRA Affinity- 1 and the integrated absorption coefficient $\left(\int \operatorname{ad} \bar{v}\right)$ was determined as per reported method. ${ }^{37} \mathrm{UV}$-visible spectra were taken on UV-visible spectrophotometer model Shimadzu UV1800 in DMSO and the molar extinction coefficient $\left(\varepsilon_{M}\right)$ as well as oscillator strength was calculated as per reported method. ${ }^{37} \mathrm{H}$-NMR spectra of the polymer solutions (prepared in $\mathrm{CDCl}_{3} 10 \mathrm{mg} \mathrm{ml}^{-1}$ ) were recorded on Bruker AC300. X-ray diffraction patterns were obtained using Philips PW 3710 powder diffractometer. Transmission electron micrographs (TEM) were recorded on Morgagni 268-D TEM, FEI, USA. Fluorescence spectra were recorded on fluorescence spectrophotometer model Horiba Fluorolog@3-11 in the solution state using $N$-methyl 2-pyrrolidinone (NMP) as solvent. The quantum yield was determined as per reported method. ${ }^{37}$ Confocal micrographs were taken on a Laser Confocal Microscope Olympus FluoView ${ }^{\mathrm{TM}}\left(\lambda_{\text {exc }}=410 \mathrm{~nm}\right)$.

\section{Cell culture and MTT assay studies}

The cancer cell line used for cell culture, Human cervical cancer cell (HeLa) was purchase from National Centre for Cell Science, Department of Biotechnology, Pune, India. The studies pertaining to cell proliferation, viability and imaging were carried out as per reported protocols. ${ }^{31,40}$

\section{Results and discussion}

\section{Determination of solubility, intrinsic viscosity and viscosity average molecular weight}

The solubility of PLU was observed to be higher in most of the organic solvents as compared to that of POPD. With increasing PLU content, the solubility of the copolymers was found to increase. The solubility of the copolymer POPD/PLU-20/80 was found to be highest among all the copolymers due to the presence of high luminol content, (ESI Table S1 $\dagger$ ). The intrinsic viscosity and viscosity average molecular weight $\left(\overline{M_{v}}\right)$ was determined using Mark-Houwink equation. ${ }^{39}$ Intrinsic viscosities were observed to be 0.90 for POPD and 0.36 for PLU while for the copolymers they were calculated to be $0.73,0.66,0.44$ for POPD/PLU-80/20, POPD/PLU-50/50, POPD/PLU-20/80 respectively (ESI Table S2 $\dagger$ ). The values of intrinsic viscosities of copolymers varied between the homopolymers. Among the copolymers, the highest $\overline{M_{v}}$ was found to be 12514 for the 


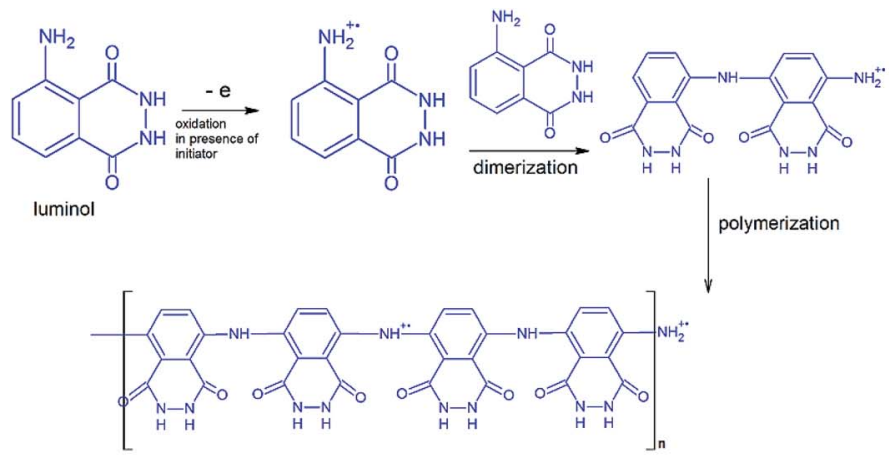

(a)

polymerization scheme of luminol homopolymer

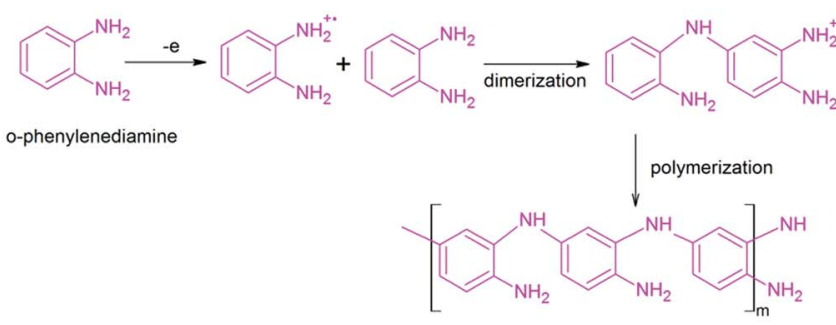

poly(o-phenylenediamine)

(b)

polymerization scheme of o-phenylenediamine homopolymer
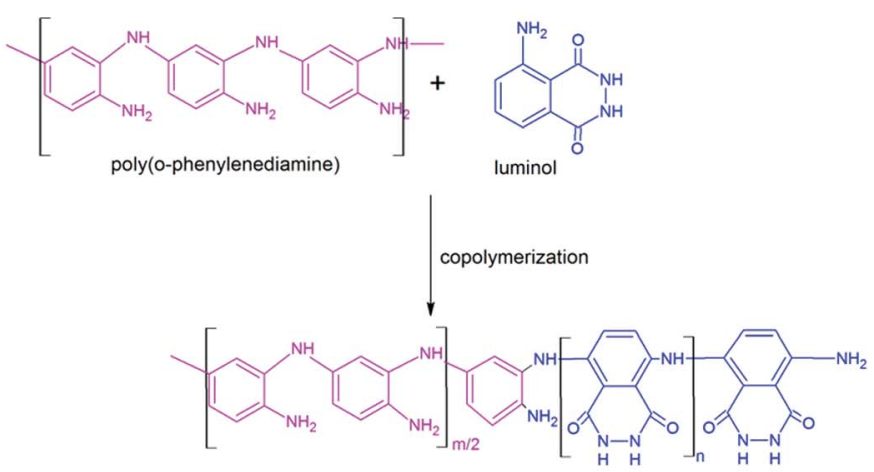

(c)

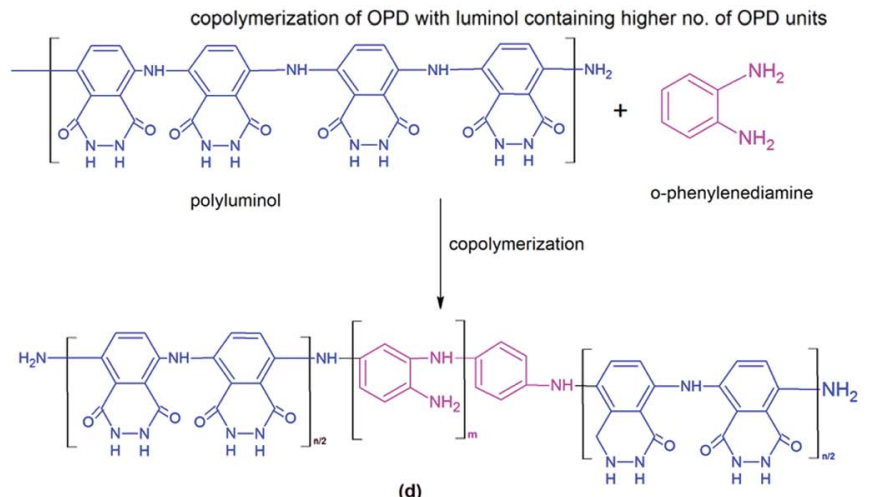

(d)

copolymerization of polyluminol with OPD containing higher no. of luminol units

Scheme 1 Synthesis of homopolymers and copolymers of POPD and PLU.

copolymer POPD/PLU-80/20 due to higher number of POPD units. Enhancement in the intrinsic viscosity was observed to be directly related to increment of molar mass but inversionally proportional to the polymerization yield (ESI Fig. S1†). 


\section{Confirmation of copolymerization by FTIR and ${ }^{1} \mathrm{H}-\mathrm{NMR}$ studies}

The ${ }^{1} \mathrm{H}$-NMR spectra of POPD, PLU and their copolymers are given in ESI, Fig. S2. $\uparrow$ The NH protons of POPD were observed at $\delta=6.9-7.9 \mathrm{ppm}$ while the NH protons of PLU were noticed between $\delta=7.4-8.5 \mathrm{ppm}$. The integrated areas of the $\mathrm{NH}$ protons of POPD (taken as 1/3) and PLU (taken as 1/4) were used for calculating their molar ratios in the copolymer. The calculated values were observed to be matching with the monomer feed ratios and therefore confirmed the copolymer composition (ESI, Table S3†).

The monomer reactivity ratios calculated from FinemanRoss parameters confirmed random copolymerization as shown in Scheme $1(\mathrm{a}-\mathrm{d}){ }^{37}$

The IR spectrum of the homopolymer POPD, Fig. 1, showed $\mathrm{N}-\mathrm{H}$ stretching vibration peak at $3134 \mathrm{~cm}^{-1}$ associated with the presence of secondary amine $(-\mathrm{NH}-) .{ }^{37}$ The peak noticed at $1610 \mathrm{~cm}^{-1}$ was correlated to imine stretching vibration while the peaks at $1402 \mathrm{~cm}^{-1}$ and $1351 \mathrm{~cm}^{-1}$ were designated to ring puckering of the quinonoid and benzenoid units respectively.

The peak observed at $1250 \mathrm{~cm}^{-1}$ was assigned to $\mathrm{CN}$ stretching vibration. The peaks noticed at $850 \mathrm{~cm}^{-1}$ and $720 \mathrm{~cm}^{-1}$ were associated with p-substituted benzene and $\mathrm{C}-\mathrm{H}$ out-of plane bending vibrations of the phenazene skeleton respectively, which confirmed the polymerization of POPD. ${ }^{37,39}$ The benzenoid : quinonoid (B:Q) ratio was calculated to be 3.46 indicating greater number of quinonoid units. The homopolymer PLU exhibited $\mathrm{NH}$ stretching vibration peak at $3132 \mathrm{~cm}^{-1}$ while the peak at $1668 \mathrm{~cm}^{-1}$ was correlated to imine stretching vibration. The peaks associated with quinonoid and benzenoid rings puckering were observed at $1402 \mathrm{~cm}^{-1}$ and $1350 \mathrm{~cm}^{-1}$ respectively and the $\mathrm{CN}$ stretching vibration peak was noticed at $1230 \mathrm{~cm}^{-1}$. The $\mathrm{B}: \mathrm{Q}$ ratio was found to be 2.94 in this case indicating greater number of quinonoid units. The IR spectrum of PLU was observed to be similar to that of POPD due to the structural similarities of the two polymers. The IR spectrum of POPD/PLU-80/20, Fig. 1, showed NH stretching vibration peak at $3154 \mathrm{~cm}^{-1}$, while the imine stretching peak appeared at $1668 \mathrm{~cm}^{-1}$. The quinonoid and benzenoid peaks were observed at $1598 \mathrm{~cm}^{-1}$ and $1402 \mathrm{~cm}^{-1}$ respectively and the

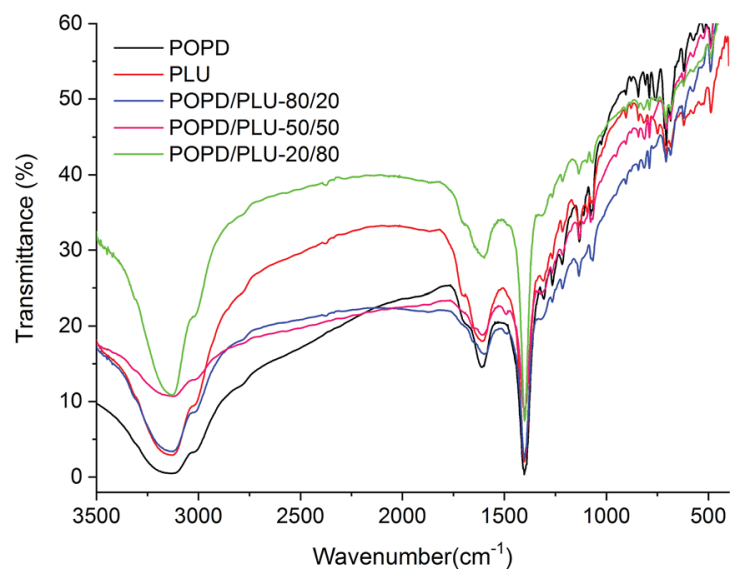

Fig. 1 FTIR spectra of homo-polymers and copolymers of POPD/PLU.
$\mathrm{B}$ : $\mathrm{Q}$ ratio was calculated to be 2.41 . The value was noticed to be intermediate of the homopolymers. The $\mathrm{NH}$ stretching vibration peak for POPD/PLU-50/50, Fig. 1, was observed at $3124 \mathrm{~cm}^{-1}$ revealing a shift of about $10 \mathrm{~cm}^{-1}$ as compared to that of pristine POPD whereas the imine stretching peak was noticed at $1633 \mathrm{~cm}^{-1}$. The B : Q ratio in this case was 1.65 which was lower than that of POPD/PLU-80/20. Similarly, POPD/PLU20/80 showed the NH stretching vibration peak at $3134 \mathrm{~cm}^{-1}$ as noticed in POPD but the imine stretching peak was noticed at $1668 \mathrm{~cm}^{-1}$ which was comparable to the one observed in PLU. The B : Q ratio was observed to be 1.17 which was lowest among all the copolymers. The NH peak area $\left(\int \mathrm{ad} \bar{v}\right)$ value was found to be 1088 for POPD and 853 for PLU. The $\int a d \bar{v}$ values for POPD/ PLU-80/20, POPD/PLU-50/50 and POPD/PLU-20/80 were calculated to be 741,568 and 380 respectively. The $\int \operatorname{ad} \bar{v}$ values were noticed to decrease with the increase in the PLU content. Likewise, the $\int \mathrm{ad} \bar{v}$ values for the imine stretching peak were observed to be 97 for POPD and 28 for PLU while for POPD/PLU80/20, POPD/PLU-50/50 and POPD/PLU-20/80, they were observed to be 44,52 and 25 respectively, showing a similar trend of decrease in the area with the increase in the PLU content as seen in case of the $\mathrm{NH}$ peak. It can therefore be concluded that copolymerization of POPD and PLU was observed to be composition dependent as the $\mathrm{NH}$ as well as the imine stretching peaks showed variation in the $\int \mathrm{ad} \bar{v}$ values which could be well correlated to the co-monomer content in the copolymer.

\section{Influence of copolymerization on morphology confirmed by XRD and TEM studies}

The XRD pattern of PLU (inset), Fig. 2, exhibited prominent peaks at $2 \theta=7.5^{\circ}, 16.3^{\circ}, 18.2^{\circ}, 19.8^{\circ}, 21.2^{\circ}, 24.3^{\circ}, 26.2^{\circ}$ and $28.3^{\circ}$ revealing a highly crystalline structure. The planes observed at (020), (010), (100), (110) and (011) revealed a pseudo orthorhombic lattice type for the polymer. ${ }^{37}$ The XRD pattern of POPD has been reported in our previous work which revealed peaks at $2 \theta=8.33^{\circ}, 18.5^{\circ}, 19.78^{\circ}, 23.5^{\circ}$ and $28.08^{\circ}$ with limited planes at (010), (110) and (011). ${ }^{37}$

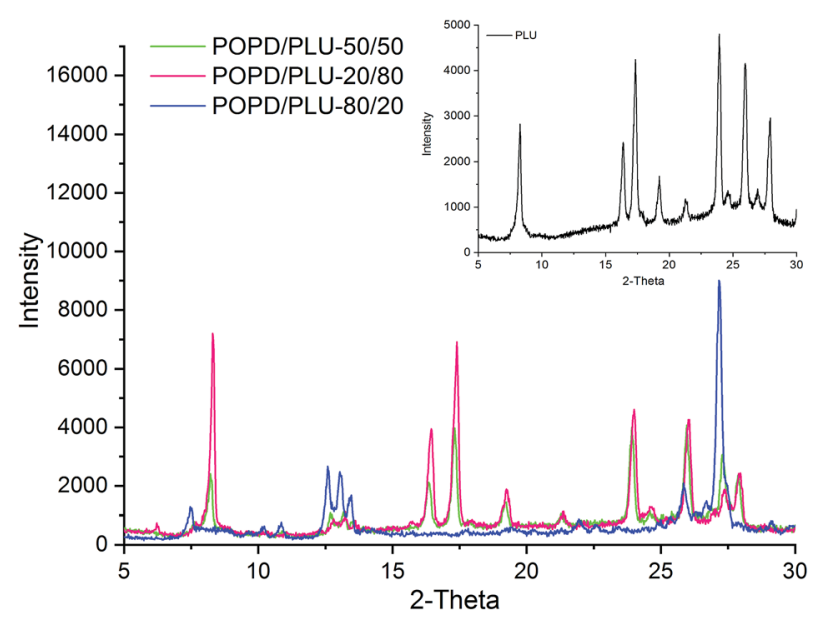

Fig. 2 XRD of PLU and POPD/PLU copolymers. 

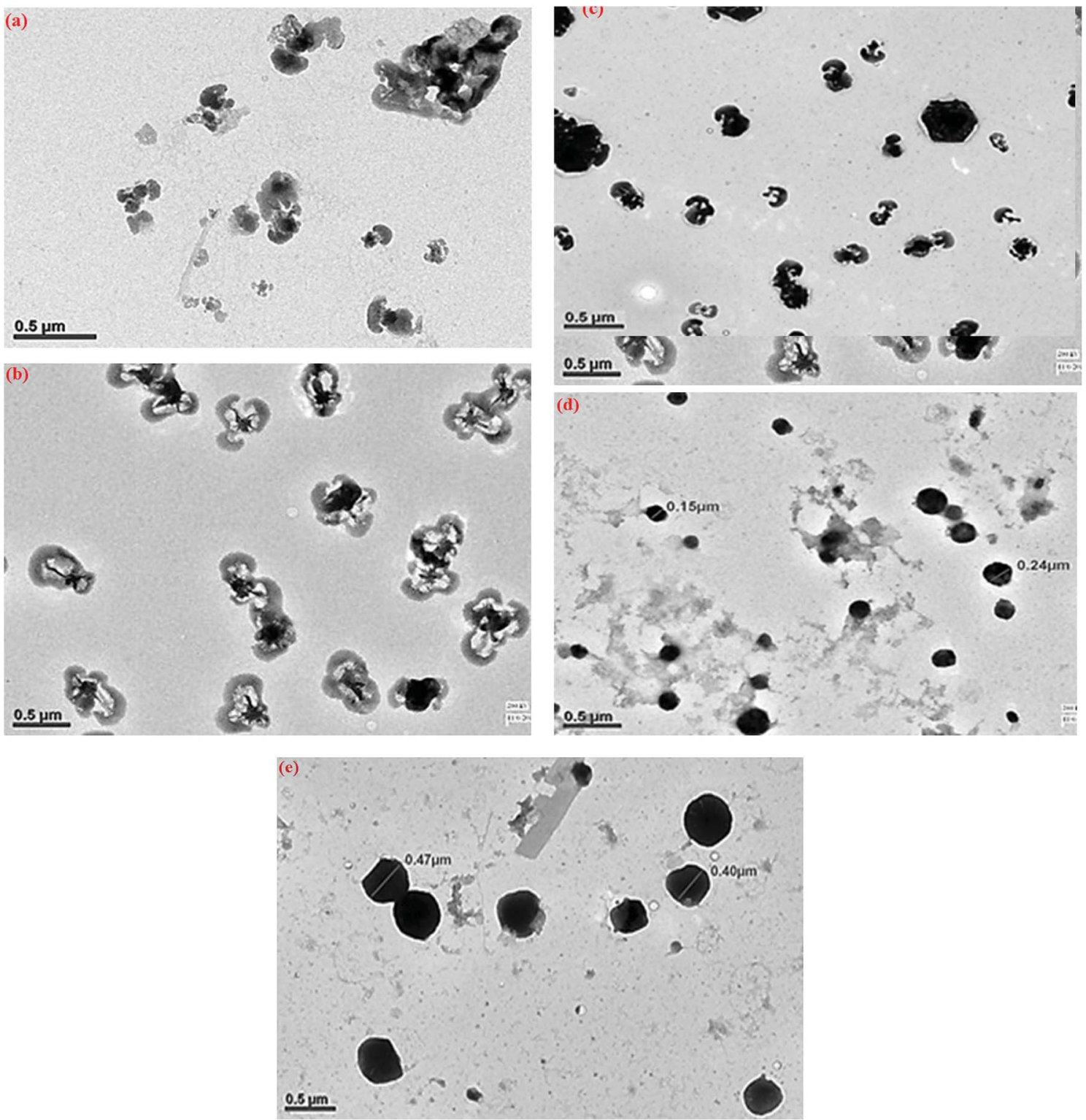

Fig. 3 TEM micrographs of (a) POPD, (b) PLU, (c) POPD/PLU-80/20, (d) POPD/PLU-50/50and (e) POPD/PLU-20/80.

The TEM of PLU, Fig. 3(a), revealed distorted cluster formation and the average size of the particles was observed to be $200 \mathrm{~nm}$. The TEM of POPD, Fig. 3(b), showed distorted seashell like morphology and the particles were observed to have a dense core surrounded by thin layer of film. The copolymer POPO/PLU-80/20, Fig. 3(c), revealed a mixed morphology of dense hexagonal and sea-shell like particles matching those observed in case of pristine POPD. The copolymer POPO/PLU50/50, Fig. 3(d), showed formation of compact spherical particles, with an average diameter of $160 \mathrm{~nm}$ while, the TEM of POPO/PLU-20/80, Fig. 3(e), showed formation of large spherical particles exhibiting an average diameter of $470 \mathrm{~nm}$. A mixed morphology was observed for the copolymers depending upon the PLU/POPD content. The copolymers revealed peaks associated with both the homopolymers along with minor variations in the $2 \theta$ values of some of the peaks. All the peaks were found to be well disposed indicating a highly organized and crystalline morphology of the copolymers. POPD/PLU-80/20 displayed peaks corresponding to PLU of lowest intensity while the intensity for the peaks corresponding to POPD was pronounced.

\section{Variation in the UV and fluorescence characteristics upon copolymerization}

The UV-visible spectra of POPD, PLU and its copolymers are depicted in Fig. 4. The peaks observed at $280 \mathrm{~nm}$ and $425 \mathrm{~nm}$ for POPD were assigned to $\pi-\pi^{*}$ transition and polaronic transition respectively as reported in our previous studies. ${ }^{37}$ The peaks associated with similar transition were observed at $270 \mathrm{~nm}$ and $375 \mathrm{~nm}$ in PLU. ${ }^{37,38}$ Interestingly, the UV-visible spectra of copolymers revealed peaks corresponding to both POPD and PLU. POPD/PLU-80/20 copolymer revealed peaks at $280 \mathrm{~nm}, 375 \mathrm{~nm}$ and $425 \mathrm{~nm}$ confirming that the individual 


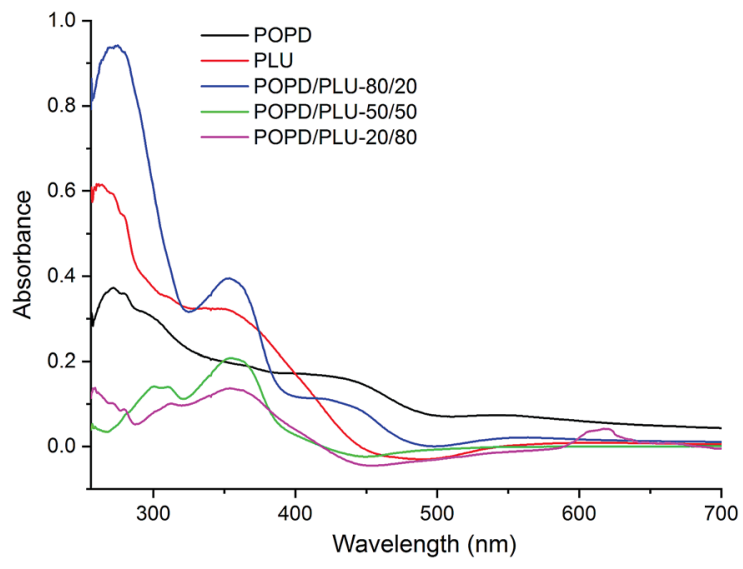

Fig. 4 UV-visible spectra of POPD, PLU and its copolymers.

electronic states of the homopolymers were retained even after copolymerization.

The intensity of the peaks corresponding to POPD was higher as compared to that of PLU confirming higher content of the former in the copolymer. Likewise, the UV spectrum of POPD/PLU-50/50 showed peaks at $300 \mathrm{~nm}, 375 \mathrm{~nm}$ confirming the presence of PLU in the copolymer. ${ }^{38}$ The peak at $425 \mathrm{~nm}$ associated with POPD was observed to be missing. Similarly in case of POPD/PLU-20/80, a broad hump was noticed at $350 \mathrm{~nm}$ and a small peak was noticed around $610 \mathrm{~nm}$. For POPD/PLU$80 / 20$, the $\int \mathrm{ad} \bar{v}$ and the $\varepsilon_{\mathrm{M}}$ values were found to be $82.32 \pm$ 0.03 and 1437 respectively while for copolymer POPD/PLU$50: 50, \int \mathrm{ad} \bar{v}$ and $\varepsilon_{\mathrm{M}}$ values were noticed to be $64.13 \pm 0.01,1077$ respectively. The $\int \mathrm{ad} \bar{v}$ and $\varepsilon_{\mathrm{M}}$ values for POPD/PLU-20/80 were found to be $40.73 \pm 0.02$ and 754 respectively. The oscillator strength was observed to be highest for pristine POPD. The oscillator strength decreased from $0.012 \pm 0.03$ to $0.0060 \pm 0.03$ as the copolymer composition of POPD/PLU varied from $80 / 20$ to $20 / 80$. Thus, the progressive change in the optical properties with the copolymer composition indicated random copolymerization (Table 1).

The fluorescence emission spectrum of POPD, Fig. 5(a), showed an intense broad peak centered at $620 \mathrm{~nm}$ upon excitation at $350 \mathrm{~nm}$ while the spectrum of PLU showed a pronounced peak at $525 \mathrm{~nm}$ at similar excitation wavelength which was attributed to $S_{1} \rightarrow S_{0}$ transition. ${ }^{37,39}$ The copolymers revealed broad emission peaks at $500 \mathrm{~nm}$ and $610 \mathrm{~nm}$.

The intensity of the peak at $610 \mathrm{~nm}$ associated with POPD was observed to increase with the increase in the number of
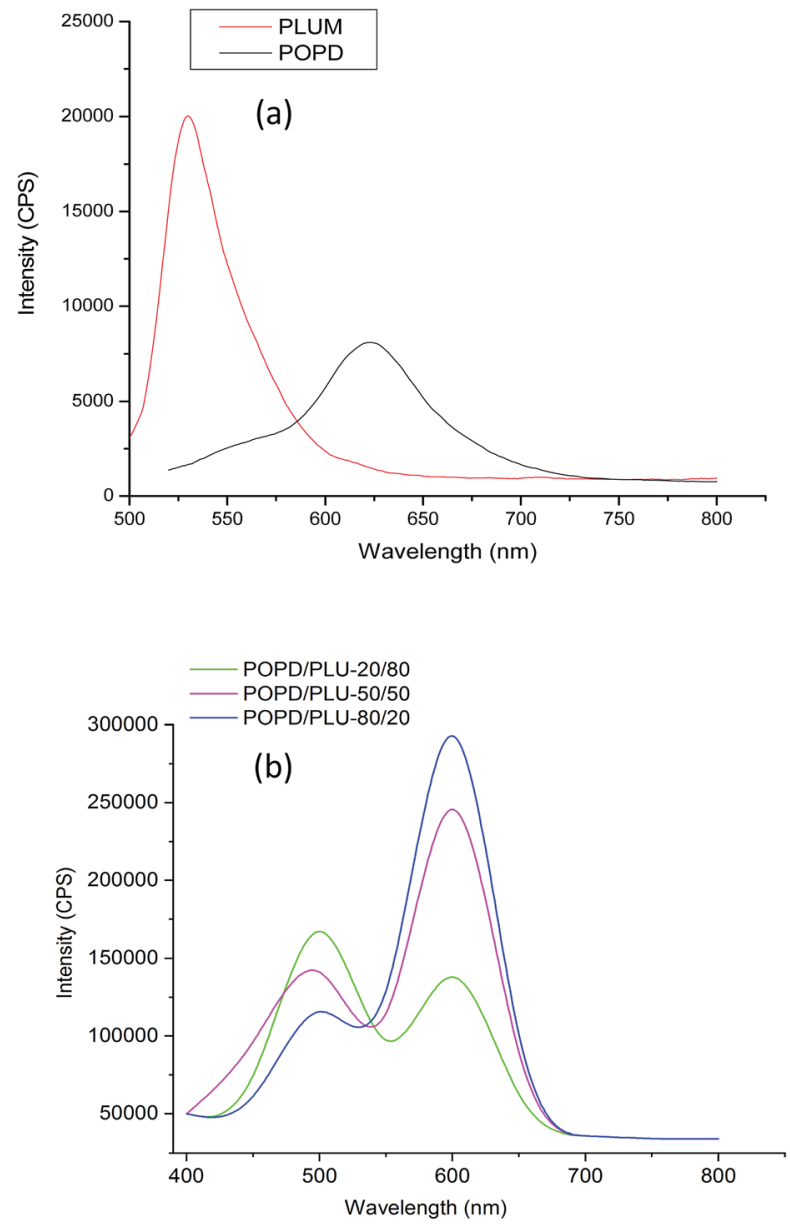

Fig. 5 Fluorescence spectra of (a) POPD, PLU (b) POPD/PLU copolymers.

POPD units in the copolymer. For POPD/PLU-80/20, the emission peak at $500 \mathrm{~nm}$ peak associated with PLU was observed to be lower, while the peak related to POPD was noticed to have highest intensity among all the copolymers due to higher number of POPD units in this copolymer. The intensity of the $500 \mathrm{~nm}$ peak was observed to increase with the increase in the number of PLU units in the copolymer. The intensity of the emission peak at $500 \mathrm{~nm}$ was observed to be $110000 \mathrm{cps}$ while the intensity for the emission peak at $610 \mathrm{~nm}$ was noticed to be 280000 cps. The copolymer POPD/PLU-50/50 revealed an emission intensity of $245000 \mathrm{cps}$ for the peak at $620 \mathrm{~nm}$ while the intensity of the emission peak at 500 was found to be

Table 1 UV data of POPD, PLU and their copolymers, data represent mean $\pm S D, n=3$

\begin{tabular}{|c|c|c|c|c|}
\hline Sample & $\lambda_{\max }(\mathrm{nm})$ & $\begin{array}{l}\int \mathrm{ad} \bar{v} \text { integrated absorption } \\
\text { coefficient, } \mathrm{cm}^{-2}\end{array}$ & $\begin{array}{l}\text { Molar extinction } \\
\text { coefficient }\left(\varepsilon_{\mathbf{M}}\right)\left(\mathrm{M}^{-1} \mathrm{~cm}^{-1}\right)\end{array}$ & Oscillator strength \\
\hline POPD & 425 & $142.46 \pm 0.02$ & 2515 & $0.022 \pm 0.02$ \\
\hline PLU & 375 & $25.93 \pm 0.03$ & 323 & $0.004 \pm 0.01$ \\
\hline POPD/PLU-80/20 & 425 & $82.32 \pm 0.03$ & 1437 & $0.012 \pm 0.03$ \\
\hline POPD/PLU-50/50 & 375 & $64.13 \pm 0.01$ & 1077 & $0.009 \pm 0.04$ \\
\hline POPD/PLU-20/80 & 610 & $40.73 \pm 0.02$ & 754 & $0.006 \pm 0.03$ \\
\hline
\end{tabular}


Table 2 Quantum yield values for homopolymers and copolymers of POPD and PLU in NMP

\begin{tabular}{|c|c|c|c|}
\hline Sample & $\lambda_{\mathrm{Em}}(\mathrm{nm})$ & $\begin{array}{l}\text { Integrated area } \\
\left(I_{\text {samp }}\right)\end{array}$ & Quantum yield $(\Phi)$ \\
\hline PLU & 525 & $3.57 \times 10^{8}$ & $0.14 \pm 0.05$ \\
\hline POPD & 620 & $2.42 \times 10^{9}$ & $0.36 \pm 0.03$ \\
\hline \multirow[t]{2}{*}{ POPD/PLU-20/80 } & 500 & $1.79 \times 10^{8}$ & $0.12 \pm 0.04$ \\
\hline & 610 & $2.34 \times 10^{7}$ & $0.22 \pm 0.06$ \\
\hline \multirow[t]{2}{*}{ POPD/PLU-50/50 } & 500 & $2.14 \times 10^{8}$ & $0.10 \pm 0.05$ \\
\hline & 610 & $2.18 \times 10^{8}$ & $0.31 \pm 0.03$ \\
\hline \multirow[t]{2}{*}{ POPD/PLU-80/20 } & 500 & $1.34 \times 10^{8}$ & $0.09 \pm 0.04$ \\
\hline & 610 & $2.32 \times 10^{9}$ & $0.34 \pm 0.02$ \\
\hline
\end{tabular}

145000 cps. The copolymer POPD/PLU-20/80, showed emission intensities of $160000 \mathrm{cps}$ and $125000 \mathrm{cps}$ for $500 \mathrm{~nm}$ and $610 \mathrm{~nm}$ peaks respectively. The $\Phi$ values were calculated by taking Rhodamine B (RhB) as reference, Table 2.

For POPD, the $\int \mathrm{ad} \bar{v}$ value of the emission peak at $620 \mathrm{~nm}$ was calculated to be $2.42 \times 10^{9}$ and the $\Phi$ value was found to be $0.36 \pm 0.03$. Similarly for PLU, the $\int \mathrm{ad} \bar{v}$ for the emission peak at $525 \mathrm{~nm}$ was calculated to be $3.57 \times 10^{8}$ and the $\Phi$ value was found to be $0.14 \pm 0.05$. The $\int \mathrm{ad} \bar{v}$ value for the emission peak at $500 \mathrm{~nm}$ in case of POPD/PLU-20/80 was found to be $1.79 \times 10^{8}$ while for the peak at $610 \mathrm{~nm}$, it was calculated to be $2.34 \times 10^{7}$. The $\Phi$ values were calculated as $0.12 \pm 0.04$ and $0.22 \pm 0.06$ corresponding to the emission peaks observed at $500 \mathrm{~nm}$ and $610 \mathrm{~nm}$ respectively. The copolymers POPD/PLU$50 / 50$ and POPD/PLU-80/20 showed $\int \mathrm{ad} \bar{v}$ values $2.14 \times 10^{8}$, $2.18 \times 10^{8}, 1.34 \times 10^{8}$ and $2.32 \times 10^{9}$ for the peaks at $500 \mathrm{~nm}$ and $610 \mathrm{~nm}$ respectively while the $\Phi$ values were found to be $0.10 \pm 0.05,0.31 \pm 0.03,0.09 \pm 0.04$ and $0.34 \pm 0.02$ respectively. The variation in the fluorescence intensity matched well with the $\int \mathrm{ad} \bar{v}$ values. As the ratio of POPD in the copolymer increased, quantum yield increased from $0.22 \pm 0.06$ to $0.34 \pm$ 0.02. Quantum yield of POPD as well as PLU were noticed to be proportional to their ratios in the copolymer. Hence, composition dependant fluorescence properties were attained upon varying the loading of POPD as well as PLU.

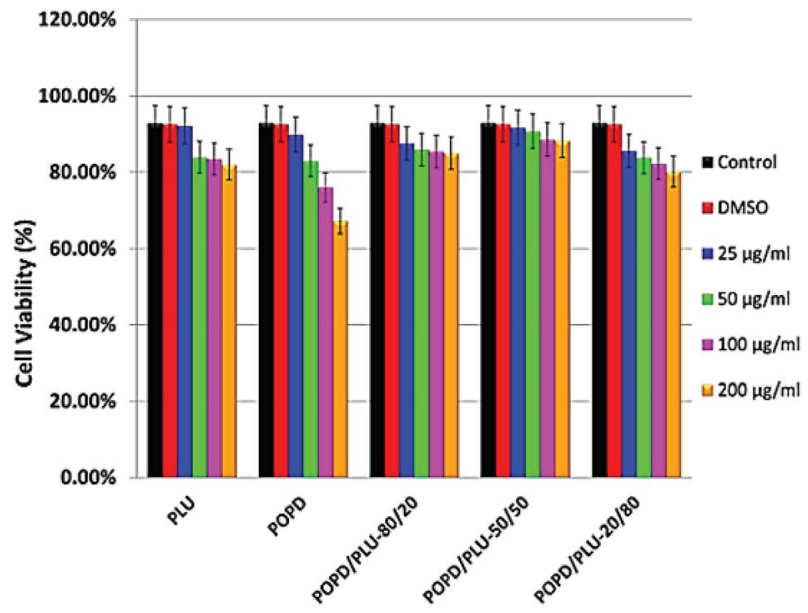

Fig. 7 Cytotoxicity analysis of POPD, PLU and its copolymers.

\section{Confocal studies}

The confocal analysis of the homopolymers and copolymers was carried out in solution state and is shown in Fig. 6. The confocal micrograph of PLU, Fig. 6(a), showed intense blue coloured particles. The fluorescence emission spectrum of PLU also revealed an emission peak around $525 \mathrm{~nm}$. POPD was noticed to emit intense red coloured tiny particles as the polymer revealed fluorescence emission peak at $620 \mathrm{~nm}$, Fig. 6(b).

The copolymer POPD/PLU/80/20, Fig. 6(c), revealed intense red coloured particles with a blue region of PLU particles. Similarly, POPD/PLU-50/50, Fig. 6(c) and POPD/PLU-20/80, Fig. 6(d) showed the presence of intense red nanoparticles which could be correlated to POPD while the PLU region was found to blue in colour. The morphology was found to consist of densely distributed aggregates of irregularly shaped fine particles of POPD dispersed in the PLU matrix. The regions of red and blue emission by the particles were found to vary with the composition of the copolymer which clearly confirmed that the homopolymers retained their photo $=$ physical properties even upon copolymerization.
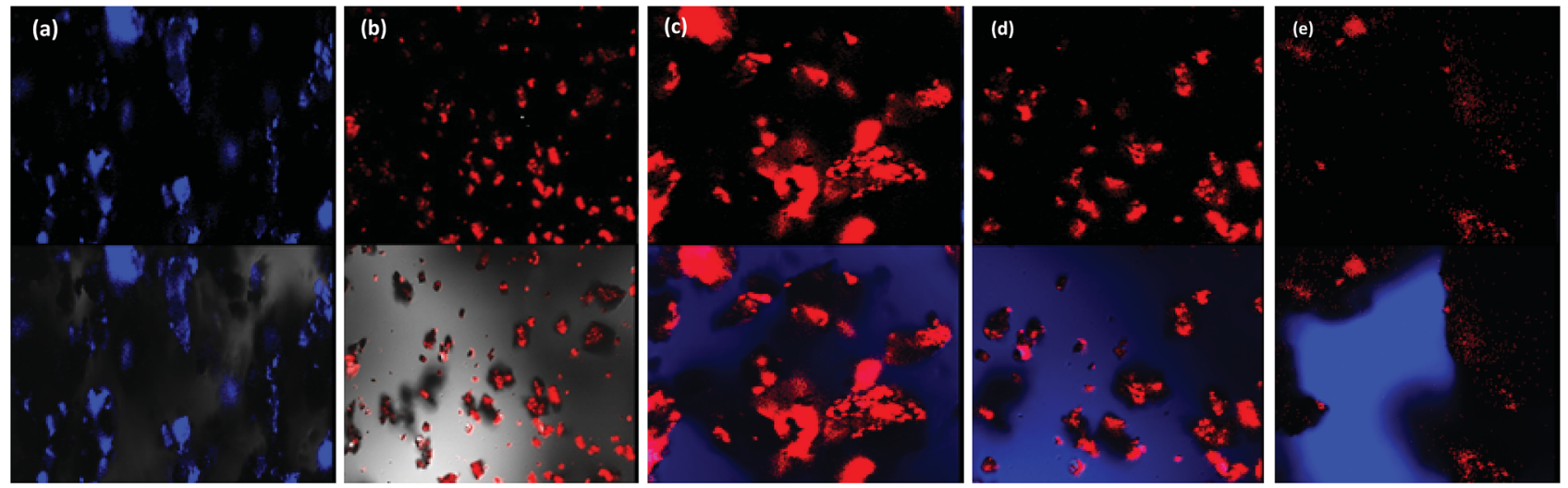

Fig. 6 Confocal micrographs of (a) PLU, (b) POPD, (c) POPD/PLU-80/20, (d) POPD/PLU-50/50, (e) POPD/PLU-20/80. 

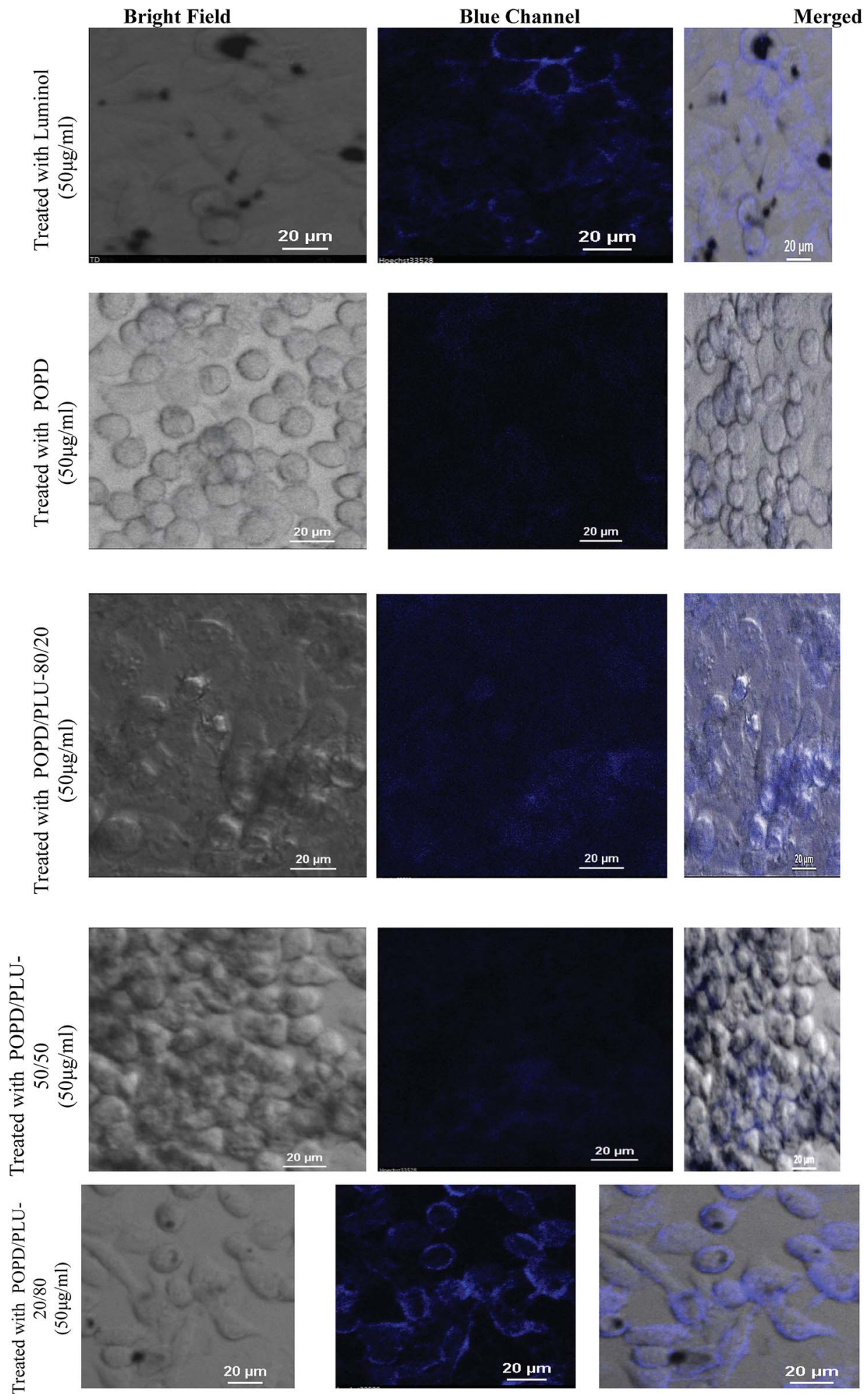

Fig. 8 Fluorescence imaging of HeLa cells incubated with the copolymers for $4 \mathrm{~h}$.

\section{Cytotoxicity and imaging of tumor cells}

HeLa cells were used to examine cellular toxicity, which included normal as well as tumor cells. The effects of homopolymers and copolymers on cell viability and proliferation were measured using the MTT assay, which is based on the reduction of the yellow tetrazolium salt MTT by metabolically active cells, resulting in purple formazan crystals. Among the homopolymers, neither PLU nor POPD revealed any toxic effect over the concentration range of $25-50 \mu \mathrm{g} \mathrm{ml}^{-1}$. Interestingly, the copolymers did not reveal any toxic effect up to the 
concentration of $200 \mu \mathrm{g} \mathrm{ml} \mathrm{g}^{-1}$, Fig. 7. Only in case of pure POPD, the concentration of $200 \mu \mathrm{g} \mathrm{ml}^{-1}$ showed to slightly block the cell viability. The cytotoxicity of the homopolymers as well as copolymers was observed to be time independent.

The copolymers were found to be non-toxic at concentrations as high as $200 \mu \mathrm{g} \mathrm{ml}^{-1}$ in all tested cell lines. The copolymer POPD/PLY-50/50 showed results quite comparable to the control group. The tumor cell lines clearly appeared to be able to tolerate exposure to copolymers through high metabolic activity during in vitro cytotoxicity tests and revealed capability of maintaining their cell proliferation and membrane integrity during exposure to higher dosages of copolymers.

The cellular uptake of POPD, PLU and its copolymers by the HeLa cells was visualized via confocal microscopic imaging, Fig. 8. The studies revealed that blue luminescence was observed by the HeLa cells treated with homopolymers as well as copolymers for a period of $4 \mathrm{~h}$, Fig. 8. Furthermore, the internalization of the pristine polymers and copolymers after $4 \mathrm{~h}$ of treatment was clearly observed in case of HeLa cells, which showed high expression levels. An intensive fluorescence signal was observed on the surface and in the cytoplasm of the HeLa cells after treatment with the PLU and POPD/PLU-20/80. In contrast, only slight fluorescence signals were observed in the HeLa cells under similar experimental conditions using POPD and copolymers containing higher POPD content. These results demonstrated the effective targeting specificity of the copolymers containing higher PLU content towards HeLa cells. To get a better insight, time-dependent confocal microscopy studies were carried out for POPD, PLU and POPD/PLU-20/80 treated HeLa cells. It was observed that the copolymer POPD/ PLU-20/80 showed a better contrast even after 24 h (ESI Fig. S3 $\dagger$ ). The time dependent confocal analysis confirmed that PLU and POPD/PLU-20/80 could be used as a novel fluorescent indicator to study the absorption, transportation processes inside the mammalian cells.

\section{Conclusion}

Microwave-assisted copolymerization of $o$-phenylenediamine with luminol was successfully carried out using different weight ratios of the two monomers. ${ }^{1} \mathrm{H}-\mathrm{NMR}$ studies confirmed random copolymerization while UV-visible and fluorescence studies showed composition dependant photo-physical properties. X-ray diffraction (XRD) and transmission electron microscopy (TEM) analyses confirmed the morphology of copolymers to be dependent on the ratio of the two monomers in the copolymer. Confocal analysis also revealed composition dependant emission of the copolymers which was found to be intense red/blue depending upon the content of POPD/PLU in the copolymer. MTT assay studies confirmed that the homopolymers as well as the copolymers were non-toxic and could be safely used for in vivo imaging of cancer cells. The confocal images of the homopolymers/copolymer modified HeLa cells revealed blue luminescence and the internalization of PLU and POPD/PLU-20/80 showed high expression levels which could be used as an efficient nanoprobe for imaging of various kinds of tumors.

\section{Conflicts of interest}

On behalf of all authors, the corresponding author states that there is no conflict of interest.

\section{Acknowledgements}

The corresponding author Dr Ufana Riaz acknowledges the Department of Science and Technology (DST)-Science and Engineering Research board DST-SERB, India vide sanction no. SB/S-1/PC-070-2013 for granting major research project. One of the co-authors Mrs Sapana Jadoun is thankful to DST-SERB for granting Senior Research Fellowship under the said project. The authors also acknowledge the SAIF Facility at All India Institute of Medical Sciences (AIIMS), New Delhi, India for TEM analysis. The authors sincerely acknowledge support of Dr Prabhat at the Advance Instrument Research Facility (AIRF), Jawaharlal Nehru University (JNU) for carrying out the live cell imaging analysis.

\section{References}

1 Z. Ge and S. Liu, Functional block copolymer assemblies responsive to tumor and intracellular microenvironments for site-specific drug delivery and enhanced imaging performance, Chem. Soc. Rev., 2013, 42, 7289-7325.

2 L. Zhu and V. P. Torchilin, Stimulus-responsive nano preparations for tumor targeting, Integr. Biol., 2013, 5, 96107.

3 P. Zhao, Q. X. u, J. Tao, Z. Jin, Y. Pan, C. Yu and Z. Yu, Near infrared quantum dots in biomedical applications: current status and future perspective, Wiley Interdiscip. Rev.: Nanomed. Nanobiotechnol., 2017, 10, e1483.

4 Y. Yang, X. Wang, G. Liao, X. Liu, Q. Chen, H. Li, L. Lu, P. Zhao and Z. Yui, iRGD-decorated red shift emissive carbon nanodots for tumor targeting fluorescence imaging, J. Colloid Interface Sci., 2018, 509, 515-521.

5 C. F. Wu and D. T. Chiu, Highly fluorescent semiconducting polymer dots for biology and medicine, Angew. Chem., Int. Ed., 2013, 52, 2-26.

6 L. L. Yang, F. F. An, Z. Liu, X. J. Zhang, M. J. Zhou, W. Li, X. J. Hao, C. S. Lee and X. H. Zhang, Ultrabright and ultrastable near-infrared dye nanoparticles for in vitro and in vivo bioimaging, Biomaterials, 2012, 33, 7803-7809.

7 U. Riaz and S. M. Ashraf, Fluorescence bioimaging with applications to chemistry, in Computational Optical Biomedical Spectroscopy and Imaging, CRC Press, 2015.

8 X. Gao, Y. Cui, R. M. Levenson, L. W. K. Chung and S. Nie, In vivo cancer targeting and imaging with semiconductor quantum dots, Nat. Biotechnol., 2004, 22, 969-976.

9 G. Libralato, E. Galdiero, A. Falang, R. Carotenuto, E. de Alteriis and M. Guida, Toxicity Effects of Functionalized Quantum Dots, Gold and Polystyrene Nanoparticles on Target Aquatic Biological Models: A Review, Molecules, 2017, 22(9), 1439-1455.

10 A. J. Hackettab, J. Malmströmb and J. T. Sejdica, Functionalization of conducting polymers for biointerface applications, Prog. Polym. Sci., 2017, 70, 18-33. 
11 D. Mantione, I. Agua, A. S. Sanchez and D. Mecerreyes, Poly(3,4-ethylenedioxythiophene) (PEDOT) Derivatives: Innovative Conductive Polymers for Bioelectronics, Polymers, 2017, 9(8), 354-385.

12 T. Cantu, K. Walsh, V. P. Pattani, A. J. Moy, J. W. Tunnell, $\mathrm{J}$. Irvin and $\mathrm{T}$. Betancourt, Conductive polymer-based nanoparticles for laser-mediated photothermal ablation of cancer: synthesis, characterization, and in vitro evaluation, Int. J. Nanomed., 2017, 12, 615-663.

$13 \mathrm{~J}$. Shan and Z. Ma, A review on amperometric immunoassays for tumor markers based on the use of hybrid materials consisting of conducting polymers and noble metal nanomaterials, Microchim. Acta, 2017, 184(4), 969-979.

14 X. Yue, Q. Zhang and Z. Dai, Near-infrared light-activatable polymeric nanoformulations for combined therapy and imaging of cancer, Adv. Drug Delivery Rev., 2017, 115, 155170.

15 B. Muthuraj, S. Mukherjee, C. R. Patra and P. K. Iyer, Amplified Fluorescence from Polyfluorene Nanoparticles with Dual State Emission and Aggregation Caused Red Shifted Emission for Live Cell Imaging and Cancer Theranostics, ACS Appl. Mater. Interfaces, 2016, 8(47), 32220-32229.

16 M. C. MacNeill, E. M. Wailes and N. H. L. Polyachenko, A Comparative Study of the Photothermal Efficiency of Electrically Conducting Poly(3,4-ethylenedioxythiophene)Based Nanomaterials with Cancer Cells, J. Nanosci. Nanotechnol., 2013, 13(6), 3784-3791.

17 D. Samanta, N. H. Nassab, A. D. McCartya and R. N. Zare, Ultra-low voltage triggered release of an anti-cancer drug from polypyrrole nanoparticles, Nanoscale, 2018, 10, 97739779.

18 Z. Long, L. Mao, M. Liu, Q. Wan, Y. Wan, X. Zhang and Y. Wei, Marrying multicomponent reactions and aggregation-induced emission (AIE): new directions for fluorescent nanoprobes, Polym. Chem., 2017, 8, 5644-5654.

19 X. Zhang, Z. Ma, Y. Yang, X. Zhang, X. Jia and Y. Wei, Fine tuning the mechano-fluorochromic properties of benzothiadiazole -cored cyano-substituted diphenylethene derivatives through D-A effect, J. Mater. Chem. C, 2014, 2, 8932-8938.

20 Y. Dong, J. W. Lam, A. Qin, J. Liu, Z. Li, B. Z. Tang, J. Sun and H. S. Kwok, Aggregation-Induced Emissions of Tetraphenylethene Derivatives and Their Utilities as Chemical Vapor Sensors and in Organic Light-Emitting Diodes, Appl. Phys. Lett., 2007, 91, 011111.

21 H. Tong, Y. Hong, Y. Dong, M. Häußler, J. W. Lam, Z. Li, Z. Guo, Z. Guo and B. Z. Tang, Fluorescent "light-up" bioprobes based on tetraphenylethylene derivatives with aggregation-induced emission characteristics, Chem. Commun., 2006, 3705-3707.

22 L. Wang, B. Xu, J. Zhang, Y. Dong, S. Wen, H. Zhang and W. Tian, Theoretical investigation of electronic structure and charge transport property of 9,10distyrylanthracene(DSA) derivatives with high solid-state luminescent efficiency, Phys. Chem. Chem. Phys., 2013, 15, 2449-2458.
23 Z. Wang, K. Ma, B. Xu, X. Li and W. Tian, A highly sensitive "turn-on" fluorescent probe for bovine serum albumin protein detection and quantification based on AIE-active distyrylanthracene derivative, Sci. China: Chem., 2013, 56, 1234-1238.

24 Y. Zhang, J. Huang, L. Kong, Y. Tian and J. Yang, Two novel AIEE-active imidazole/ $\alpha$-cyanostilbene derivatives: photophysical properties, reversible fluorescence switching, and detection of explosives, CrystEngComm, 2018, 20, 1237-1244. 25 X. He, G.-B. Zhang, Z.-X. Chi, P.-F. Dai, J.-Y. Huang and J.-X. Yang, Terpyridine functionalized $\alpha$-cyanostilbene derivative as excellent fluorescence and naked eyes $\mathrm{Fe}^{2+}$ probe in aqueous environment, Chem. Pap., 2017, 71(11), 2209-2221.

26 Y. Yang, Q. Chen, S. Li, W. Ma, G. Yao, F. Ren, Z. Cai, P. Zhao, G. Liao, J. Xiong and Z. Yu, iRGD-Mediated and EnzymeInduced Precise Targeting and Retention of Gold Nanoparticles for the Enhanced Imaging and Treatment of Breast Cancer, J. Biomed. Nanotechnol., 2018, 14, 1396-1408. 27 L. Esser, N. P. Truong, B. Karagoz, B. A. Moffat, C. Boyer, J. F. Quinn, M. R. Whittaker and T. P. Davis, GadoliniumFunctionalized Nanoparticles for Application as Magnetic Resonance Imaging Contrast Agents via PolymerizationInduced Self-Assembly, Polym. Chem., 2016, 7, 7325-7337.

28 S. Y. Khor, M. N. Vu, E. H. Pilkington, A. P. R. Johnston, M. R. Whittaker, J. F. Quinn, N. P. Truong and T. P. Davis, Elucidating the Influences of Size, Surface Chemistry, and Dynamic Flow on Cellular Association of Nanoparticles Made by Polymerization-Induced Self-Assembly, Small, 2018, 14(34), e1801702.

29 E. T. Garrett, Y. Pei and A. B. Lowe, Microwave-assisted synthesis of block copolymer nanoparticles via RAFT with polymerization induced self-assembly in methanol, Polym. Chem., , 2016, 7, 297-301.

30 F. Fan, C. Cai, L. Gao, J. Li, P. Zhang, G. Li, C. Li and G. Yu, Microwave-assisted synthesis of glycopolymers by ringopening metathesis polymerization (ROMP) in an emulsion system, Polym. Chem., 2017, 8, 6709-6719.

31 U. Riaz, S. Jadoun, P. Kumar, M. Arish, A. Rub and S. M. Ashraf, Influence of Luminol Doping of Poly $(o-$ phenylenediamine) on the Spectral, Morphological, and Fluorescent properties: A Potential Fluorescent Marker for Early detection and Diagnosis of Leishmania donovani, ACS Appl. Mater. Interfaces, 2017, 9(38), 33159-33168.

32 S. Jadoun, V. Sharma, S. M. Ashraf and U. Riaz, Sonolytic doping of poly(1-naphthylamine) with luminol: influence on spectral, morphological and fluorescent characteristics, Colloid Polym. Sci., 2017, 295(4), 715-724.

33 Y. P. Dong, J. Wang, Y. Peng and J. J. Zhu, A Novel Aptasensor for Lysozyme based on Electrogenerated Chemiluminescence Resonance Energy Transfer Between Luminol and Silicon Quantum Dots, Biosens. Bioelectron., 2017, 94, 530-535.

34 P. Khan, D. Idrees, M. A. Moxley, J. A. Corbett, F. Ahmad, G. Figura, W. S. Sly, A. Waheed and M. I. Hassan, Luminol Based Chemiluminescent Signals: Clinical and Non- 
Clinical Application and Future Uses, Appl. Biochem. Biotechnol., 2014, 173(2), 333-355.

35 S. Samanta, P. Roy and P. Kar, Synthesis of poly(o-phenylenediamine) nanofiber with novel structure and properties, Polym. Adv. Technol., 2017, 28(7), 797-804.

36 J. L. O. Martínez, B. I. F. Mancilla, A. V. Rios and E. A. Z. Contreras, Poly(ortho-phenylenediamine-co-aniline) based copolymer with improved capacitance, J. Power Sources, 2017, 366, 233-240.

37 S. Jadoun, S. M. Ashraf and U. Riaz, Tuning the spectral, thermal and fluorescent properties of conjugated polymers via random copolymerization of hole transporting monomers, RSC Adv., 2017, 7, 32757-32768.
38 S. Jadoun, S. M. Ashraf and U. Riaz, Microwave-assisted synthesis of copolymers of luminol with anisidine: Effect on spectral, thermal and fluorescence characteristics, Polym. Adv. Technol., 2018, 29(2), 1007-1017.

39 U. Riaz, S. M. Ashraf, S. Aleem, V. Budhiraja and S. Jadoun, Microwave-Assisted Green Synthesis of Some Nanoconjugated Copolymers: Characterisation and Fluorescence Quenching Studies with Bovine Serum Albumin, New J. Chem., 2016, 40, 4643-4653.

40 R. Kumar and A. B. Tiku, Galangin induces cell death by modulating the expression of glyoxalase-1 and Nrf-2 in HeLa cells, Chem.-Biol. Interact., 2018, 279(5), 1-9. 